\title{
Electrocardiographic features of immune checkpoint inhibitor associated myocarditis
}

\author{
Daniel A Zlotoff (D) , ${ }^{1}$ Malek Z O Hassan, ${ }^{2}$ Amna Zafar, ${ }^{2}$ Raza M Alvi, ${ }^{2}$ \\ Magid Awadalla, ${ }^{2}$ Syed S Mahmood, ${ }^{3}$ Lili Zhang, ${ }^{4}$ Carol L Chen, ${ }^{5}$ \\ Stephane Ederhy, ${ }_{6}^{6}$ Ana Barac, ${ }^{7}$ Dahlia Banerji, ${ }^{2}$ Maeve Jones-O'Connor, ${ }^{1}$ \\ Sean P Murphy, ${ }^{1}$ Merna Armanious, ${ }^{8}$ Brian J Forrestal, ${ }^{7}$ Michael C Kirchberger, ${ }^{9}$ \\ Otavio R Coelho-Filho, ${ }^{10}$ Muhammad A Rizvi, ${ }^{11}$ Gagan Sahni, ${ }^{12}$ Anant Mandawat, ${ }^{13}$ \\ Carlo G Tocchetti, ${ }^{14}$ Sarah Hartmann, ${ }^{2}$ Hannah K Gilman, ${ }^{2}$ \\ Eduardo Zatarain-Nicolás, ${ }^{15}$ Michael Mahmoudi, ${ }^{16}$ Dipti Gupta, ${ }^{5}$ Ryan Sullivan, ${ }^{17}$ \\ Sarju Ganatra, ${ }^{18}$ Eric H Yang, ${ }^{19}$ Lucie M Heinzerling, ${ }^{20}$ Franck Thuny (D),${ }^{21}$ \\ Leyre Zubiri, ${ }^{17}$ Kerry L Reynolds, ${ }^{17}$ Justine V Cohen, ${ }^{22}$ Alexander R Lyon, ${ }^{23}$ \\ John Groarke, ${ }^{24}$ Paaladinesh Thavendiranathan, ${ }^{25}$ Anju Nohria, ${ }^{24}$ \\ Michael G Fradley, ${ }^{8}$ Tomas G Neilan $^{1,2}$
}

To cite: Zlotoff DA, Hassan MZO, Zafar A, et al. Electrocardiographic features of immune checkpoint inhibitor associated myocarditis. Journal for ImmunoTherapy of Cancer 2021;9:e002007. doi:10.1136/ jitc-2020-002007

- Additional material is published online only. To view, please visit the journal online (http://dx.doi.org/10.1136/jitc2020-002007).

Accepted 24 January 2021

Check for updates

(C) Author(s) (or their employer(s)) 2021. Re-use permitted under CC BY-NC. No commercial re-use. See rights and permissions. Published by BMJ.

For numbered affiliations see end of article.

Correspondence to

Tomas G Neilan;

tneilan@mgh.harvard.edu

\section{ABSTRACT}

Background Myocarditis is a highly morbid complication of immune checkpoint inhibitor (ICI) use that remains inadequately characterized. The QRS duration and the QTC interval are standardized electrocardiographic measures that are prolonged in other cardiac conditions; however, there are no data on their utility in ICl myocarditis. Methods From an international registry, ECG parameters were compared between 140 myocarditis cases and 179 controls across multiple time points (pre-ICl, on $\mathrm{ICl}$ prior to myocarditis, and at the time of myocarditis). The association between ECG values and major adverse cardiac events (MACE) was also tested.

Results Both the QRS duration and QTC interval were similar between cases and controls prior to myocarditis. When compared with controls on an ICl $(93 \pm 19 \mathrm{~ms})$ or to baseline prior to myocarditis $(97 \pm 19 \mathrm{~ms})$, the QRS duration prolonged with myocarditis $(110 \pm 22 \mathrm{~ms}, \mathrm{p}<0.001$ and $p=0.009$, respectively). In contrast, the QTc interval at the time of myocarditis $(435 \pm 39 \mathrm{~ms})$ was not increased compared with pre-myocarditis baseline $(422 \pm 27 \mathrm{~ms}$, $\mathrm{p}=0.42)$. A prolonged $\mathrm{QRS}$ duration conferred an increased risk of subsequent MACE (HR $3.28,95 \% \mathrm{Cl} 1.98$ to 5.62, $\mathrm{p}<0.001$ ). After adjustment, each $10 \mathrm{~ms}$ increase in the QRS duration conferred a 1.3-fold increase in the odds of MACE $(95 \% \mathrm{Cl} 1.07$ to $1.61, \mathrm{p}=0.011)$. Conversely, there was no association between the QTc interval and MACE among men (HR 1.33, $95 \% \mathrm{Cl} 0.70$ to $2.53, \mathrm{p}=0.38$ ) or women (HR 1.48, 95\% Cl 0.61 to $3.58, \mathrm{p}=0.39$ ). Conclusions The QRS duration is increased in ICI myocarditis and is associated with increased MACE risk. Use of this widely available ECG parameter may aid in ICI myocarditis diagnosis and risk-stratification.

\section{BACKGROUND}

Since the introduction of ipilimumab in 2011, immune checkpoint inhibitors (ICIs) have improved oncological care, offering new therapeutic options for a variety of cancers. ${ }^{1}$ Through activation of the immune system, ICIs can enhance antitumor activity but may also lead to immune-related adverse events (irAEs)..$^{2}{ }^{3}$ Myocarditis is an uncommon irAE. $^{4}{ }^{5}$ The incidence of ICI-associated myocarditis is unclear, with estimates ranging from $0.09 \%$ to $1.1 \% .^{36-10}$ There are several potential cardiac adverse effects associated with ICIs but the focus on myocarditis is due to the morbidity and mortality associated with that diagnosis. ${ }^{1112}$ Specifically, data from multiple groups report a mortality ranging from $17 \%$ to $50 \% .^{36713}$ In comparison, the mortality of non-ICI myocarditis is far less than $5 \% .{ }^{1415}$ Therefore, there is a significant need for improved diagnostic and riskstratification methods in ICI myocarditis, as well as newly recognized cardiac toxicities related to other forms of immune therapy for cancer. ${ }^{1617}$

The QRS duration and the QTc interval are standardized measures routinely available from a 12-lead ECG. The QRS duration corresponds to ventricular depolarization, while the QTc interval predominantly represents ventricular repolarization. Monitoring of the QRS duration is employed in the care of patients who are at risk of developing arrhythmias and heart failure. ${ }^{1819}$ Measurement of the QTc interval is also routinely used in both oncology trials and routine oncology care. ${ }^{20}$ However, there are no data on the utility of measurement of 
the QRS duration and the QTc interval among patients with ICI myocarditis. Therefore, the goal of this study was to evaluate whether the QRS duration and QTc interval increase with ICI myocarditis and whether these parameters associate with the development of subsequent adverse cardiac events.

\section{METHODS \\ Patients}

Data on myocarditis cases $(n=140)$ were obtained from a 23-center international registry specifically designed for collating cases of ICI myocarditis. ${ }^{5} 721$ Cases were included between November 2013 and April 2019. Controls $(n=179)$ were selected patients from a Massachusetts General Hospital registry of patients treated with an ICI during the same time interval for whom ECG data were available. Controls were not selected to match on any parameter with the cases.

\section{ECG data}

ECGs were obtained at various timepoints relative to ICI initiation and to the development of myocarditis (among cases). The acquisition of an ECG was not protocol-specified and was performed at the discretion of the medical care team. ECGs were recorded using the preferred system at each registry site and the parameters were derived automatically by those systems. Forty randomly selected ECGs (20 from controls and 20 from myocarditis cases) were also manually interpreted by a reader (DAZ) blinded to the case/control identity and the automated measurements. These manually measured values were then compared with those which had been automatically derived. The primary measures of interest included the PR interval, QRS duration, and the QTc interval. The PR interval represents the time delay between atrial depolarization and ventricular depolarization and was included in the analysis to assess which measures of intracardiac conduction were affected in ICI myocarditis. QT interval correction for heart rate was calculated using the Fridericia formula ${ }^{22}$ as this is more accurate than the commonly used Bazett formula and considered the most appropriate strategy by the US Food and Drug Administration. ${ }^{23}{ }^{24}$ Based on standardized criteria, a QRS duration greater than $110 \mathrm{~ms}$ was considered prolonged. ${ }^{25}$ Similarly, based on standardized criteria, a QTc interval greater than $450 \mathrm{~ms}$ in men and $460 \mathrm{~ms}$ in women was considered prolonged. ${ }^{26}$

\section{Clinical data}

Clinical data of interest obtained retrospectively from electronic medical records included patient demographics, medications, prior medical conditions, lab values, and cardiovascular risk factors. Data relevant to cancer included the cancer type, prior cancer therapies, and specific ICI treatments.

\section{Definitions and outcomes of interest}

Myocarditis was diagnosed through one of the following standardized approaches: (1) histopathological features on endomyocardial biopsy or autopsy or (2) a clinically accepted scoring system designed for suspected myocarditis that uses clinical findings, biomarkers, and imaging features. ${ }^{27}$ This latter clinical diagnostic algorithm relies on the presence of one or more clinical features (chest pain, heart failure, arrhythmias, or cardiogenic shock) plus one or more diagnostic findings (ECG abnormalities, elevated troponin levels, functional or structural abnormalities on cardiac imaging, or evidence of edema or late gadolinium enhancement on cardiac MRI) occurring in the absence of significant coronary disease or known alternative causes. ${ }^{27}$ This standardized scoring system was devised because an endomyocardial biopsy is typically performed in less than $15 \%$ of myocarditis cases. ${ }^{28}$ The main outcome of interest was major adverse cardiac events (MACE), which was a composite of cardiovascular death, ${ }^{29}$ cardiac arrest, ${ }^{30}$ cardiogenic shock, ${ }^{31}$ and hemodynamically significant complete heart block. ${ }^{7}$

\section{Statistical analysis}

Continuous variables were summarized as either the mean $\pm \mathrm{SD}$ or as the median and IQR, as appropriate, and categorical variables were presented as percentages. Comparisons between myocarditis cases and controls were made with Fisher's exact test for categorical variables or with the Wilcoxon rank sum test for continuous variables (age and body mass index (BMI), both of which demonstrated non-Gaussian distribution). Comparisons of ECG parameters by case and ICI exposure status used the Kruskal-Wallis test with Dunn's correction for multiple comparisons. The relationship between measured ECG parameters and left ventricular ejection fraction (EF) or left ventricular end-diastolic volume (LVEDV) was analyzed with simple linear regression and the associations were tested with the Pearson correlation coefficient. HRs for the association of dichotomized ECG parameters with MACE were determined using Cox proportional hazard models with follow-up time used as the time scale. The assumption of proportionality was verified by the method of Therneau and Grambsch. ${ }^{32}$ Kaplan-Meier curves and the log-rank test were used to analyze the relationship between QRS duration or QTc interval and MACE-free survival. The $\chi^{2}$ test was used to compare the frequencies of MACE component endpoints among myocarditis cases and subgroups. Multivariable logistic regression was used to address the relationship between multiple covariates, including ECG parameters at the time of myocarditis, and MACE risk. All statistical tests were two-sided and $p<0.05$ was considered significant. Statistical analysis was performed using R V.3.5.1 (R Foundation for Statistical Computing, Vienna, Austria) and SPSS V.26 (IBM Corporation). 


\section{RESULTS}

\section{Patient characteristics}

One hundred and forty patients with ICI myocarditis and 179 controls were included in the analysis. The median time between ICI initiation and myocarditis diagnosis was 58 days, and 91 cases $(65.0 \%)$ occurred within the first 90 days of ICI therapy. Of the 140 cases, $70(50.0 \%)$ were diagnosed based on clinical criteria, 58 patients (41.4\%) were diagnosed by pathology from an endomyocardial biopsy, and 12 patients (8.6\%) were diagnosed based on autopsy pathology. The median duration of follow-up for myocarditis cases was 69 days (IQR 19-153). Myocarditis patients had a higher BMI than controls $(28 \pm 6.1$ vs 26 $\pm 5.8, \mathrm{p}=0.002$ ); otherwise, cases and controls had similar rates of cardiovascular risk factors (table 1). The most common cancer in both groups was melanoma. Renal cell cancer was more common among myocarditis cases $(8.6 \%$ vs $2.2 \%, \mathrm{p}=0.02)$. The use of atezolizumab was higher among cases $(7.1 \%$ vs $1.7 \%, \mathrm{p}=0.02)$. There was a lower frequency of anti-programmed cell death 1 (PD-1) therapy use in the myocarditis group $(87.1 \%$ vs $96.1 \%$, $\mathrm{p}=0.005$ ); conversely, there was a greater frequency of anti-programmed death ligand 1 (PD-L1) therapy use in the myocarditis group ( $11.4 \%$ vs $2.2 \%, \mathrm{p}=0.001)$. The use of ipilimumab/nivolumab combination therapy was also more common in the myocarditis group $(25.7 \%$ vs $11.2 \%$, $\mathrm{p}=0.001)$. The rates of pneumonitis $(24.3 \%$ vs $12.3 \%$, $\mathrm{p}=0.007)$ and neurological adverse events $(8.6 \%$ vs $2.2 \%$, $\mathrm{p}=0.02$ ) were higher in the myocarditis group.

\section{ECG characteristics}

We compared computer-derived ECG parameters between controls and myocarditis cases at three different time-points: prior to initiation of ICI ("pre-ICI"), after the initiation of ICI but prior to development of myocarditis ("on-ICI"), and at the time of presentation with myocarditis. For validation of this approach, we also manually measured the parameters from 40 random ECGs (20 control and 20 cases) and found these values to be similar to the computer-derived values (online supplemental file 1). The median time intervals between pre-ICI baseline ECG and initiation of ICI were 103 days (IQR 24-215) and 82 days (IQR 35-172), controls and cases, respectively. The median time intervals between initiation of ICI and on-ICI ECG were 165 days (IQR 41-379) and 44 days (IQR 11-152), controls and cases, respectively. The pre-ICI baseline PR interval, QRS duration, and QTc (corrected for heart rate with the Fridericia formula, subsequently designated QTc-F) interval were similar between controls and cases (figure 1A-C). The on-ICI (prior to development of myocarditis for cases) PR interval, QRS duration, and QTc-F did not change from baseline and were similar between cases and controls. Additionally, among cases prior to the development of myocarditis, there was no increase in these parameters between the pre-ICI values and the on-ICI values. With the development of myocarditis, the PR interval did not increase. In contrast, the QRS duration at the time of myocarditis $(110 \pm 22 \mathrm{~ms})$ did increase and was greater than the QRS duration of cases pre-ICI $(99 \pm 20 \mathrm{~ms}, \mathrm{p}=0.001)$, controls on-ICI $(93 \pm 19 \mathrm{~ms}$, $\mathrm{p}<0.001)$, and cases on-ICI prior to the development of myocarditis $(97 \pm 19, \mathrm{p}=0.009)$. Cases were stratified by QRS duration using the cut-off of $110 \mathrm{~ms}$, the upper limit of normal in adults. ${ }^{25} \mathrm{~A}$ normal QRS duration of $\leq 110 \mathrm{~ms}$ was observed in 74 cases $(52.8 \%)$, while $66(47.1 \%)$ had a prolonged QRS duration $>110 \mathrm{~ms}$. A similar testing strategy was adopted for the QTc-F interval. The QTc-F interval at time of myocarditis $(435 \pm 39 \mathrm{~ms})$ was increased when compared with controls on ICI $(419 \pm 28 \mathrm{~ms}, \mathrm{p}=0.02)$ but not when compared with values from cases prior to the development of myocarditis $(422 \pm 27 \mathrm{~ms}, \mathrm{p}=0.42)$. Of 99 male cases, $29(29.3 \%)$ had a normal QTc-F $\leq 450 \mathrm{~ms}$, while $70(70.7 \%)$ had a prolonged QTc-F $>450 \mathrm{~ms}$. Among women with myocarditis, $25(61.0 \%)$ had a normal QTc-F $\leq 460 \mathrm{~ms}$, while $16(39.0 \%)$ had a prolonged QTc$\mathrm{F}>460 \mathrm{~ms}$. We also analyzed the QTc interval across time points using the Bazett formula to correct for heart rate (subsequently designated QTc-B). With this approach, the QTc-B interval at the time of myocarditis $(460 \pm 36 \mathrm{~ms})$ was increased when compared with either on-ICI controls $(440 \pm 28 \mathrm{~ms}, \quad \mathrm{p}<0.001)$ or on-ICI cases $(440 \pm 29 \mathrm{~ms}$, $\mathrm{p}=0.007$ ) (online supplemental file 2 ).

\section{Sensitivity of QRS duration for the diagnosis of ICI myocarditis} Given the significantly prolonged QRS duration observed during myocarditis, the sensitivity and specificity of QRS duration towards myocarditis diagnosis was determined. Applying a cut-off of $>110 \mathrm{~ms}$ achieved a sensitivity of $48.6 \%$ and specificity of $87.0 \%$ for the diagnosis of myocarditis; the application of a higher cut-off of $>130 \mathrm{~ms}$ yielded a sensitivity of $16.4 \%$ and a specificity of $92.6 \%$ (online supplemental table 1). A receiver-operating characteristic curve applying QRS duration towards myocarditis diagnosis had an area under the curve of 0.73 (online supplemental figure 3). Using the change in QRS duration ( $\triangle$ QRS; equal to the QRS duration at time of myocarditis minus the QRS duration at baseline prior to myocarditis) yielded a sensitivity of $51.7 \%$ when applying a diagnostic threshold of $>10 \mathrm{~ms}$; this decreased to $31.0 \%$ and $21.6 \%$ for $>20 \mathrm{~ms}$ and $>30 \mathrm{~ms}$, respectively.

\section{Association of ECG parameters with left ventricular size and function}

We analyzed the relationship between the measured ECG parameters and left ventricular EF and LVEDV among myocarditis cases (figure 2A-C). The QRS duration was not related to either echocardiographic EF $(r=-0.10$, $95 \%$ CI -0.27 to $0.07, \mathrm{p}=0.25)$ or MRI-derived EF ( $\mathrm{r}=-0.095$, $95 \%$ CI -0.29 to $0.11, \mathrm{p}=0.35$ ), but was directly related to the LVEDV ( $\mathrm{r}=0.32,95 \%$ CI 0.13 to $0.49, \mathrm{p}=0.001$ ). The QTc-F interval was inversely associated with the echocardiographic EF ( $r=-0.20,95 \%$ CI -0.36 to $-0.02, p=0.026)$ and the MRI-derived EF ( $\mathrm{r}=-0.20,95 \% \mathrm{CI}-0.039$ to -0.01 , $\mathrm{p}=0.044)$, but was not related to the LVEDV ( $\mathrm{r}=0.16$, $95 \%$ CI -0.04 to $0.35, \mathrm{p}=0.12$ ). 
Table 1 Baseline characteristics

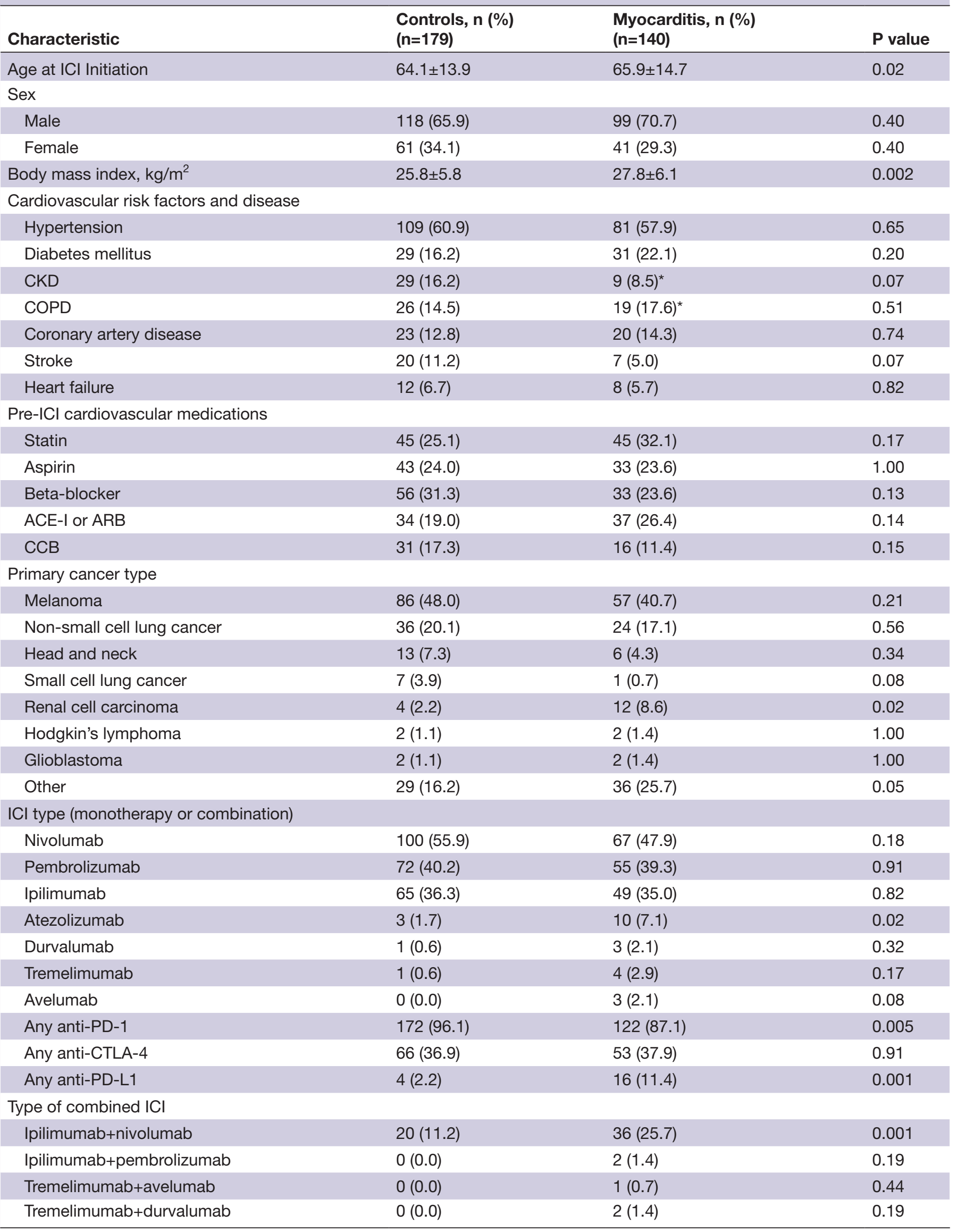


Table 1 Continued

\begin{tabular}{|c|c|c|c|}
\hline Characteristic & $\begin{array}{l}\text { Controls, n (\%) } \\
(n=179)\end{array}$ & $\begin{array}{l}\text { Myocarditis, } n(\%) \\
(n=140)\end{array}$ & $P$ value \\
\hline \multicolumn{4}{|l|}{ Other adverse events } \\
\hline Colitis & $24(13.4)$ & 13 (9.3) & 0.29 \\
\hline Pneumonitis & $22(12.3)$ & $34(24.3)$ & 0.007 \\
\hline Hepatitis & $10(5.6)$ & $16(11.4)$ & 0.07 \\
\hline Dermatitis & $5(2.8)$ & $10(7.1)$ & 0.11 \\
\hline Neurological & $4(2.2)$ & $12(8.6)$ & 0.02 \\
\hline Other & $6(3.4)$ & $7(5.0)$ & 0.57 \\
\hline \multicolumn{4}{|l|}{ Prior cancer therapies } \\
\hline
\end{tabular}

Values are $\mathrm{n}(\%)$ or mean \pm SD (for age and BMI only).

${ }^{*}$ Certain patients with incomplete data were not included in analysis for some variables in the myocarditis group; percentages were derived from lower denominators in these rows (106 for CKD and 108 for COPD).

ACE-I, Angiotensin converting enzyme inhibitor; ARB, angiotensin receptor blocker; BMI, body mass index; CCB, calcium channel blocker; CKD, chronic kidney disease; COPD, chronic obstructive pulmonary disease; ICl, immune checkpoint inhibitor; PD-1, programmed cell death 1; PD-L1, programmed death ligand 1; VEGF, vascular endothelial growth factor.

\section{Association of QRS duration with MACE}

Overall, 69 of the 140 cases (49\%) experienced a MACE. The median time to a MACE was 34 days (IQR 12-103) and $68 \%$ of MACE occurred within the first 90 days after diagnosis of myocarditis. A non-sinus rhythm at presentation with myocarditis was associated with an HR for MACE of 1.94 relative to sinus rhythm at presentation $(95 \% \mathrm{CI}$ 1.27 to 3.43 , log-rank $\mathrm{p}=0.01$ ) (online supplemental figure 4). Using an unadjusted Cox proportional hazard model, a QRS duration $>110 \mathrm{~ms}$ was associated with a HR for MACE of 3.28 relative to a QRS duration $\leq 110 \mathrm{~ms}$ $(95 \%$ CI 1.98 to $5.62, \mathrm{p}<0.001$ ) (figure $3 \mathrm{~A})$. Increased QRS duration at the time of myocarditis was associated with increased MACE risk after adjustment for age, sex, and cardiovascular comorbidities (table 2). Specifically, an increase in the QRS duration of $10 \mathrm{~ms}$ conferred a 1.30 -fold increase in the odds of MACE (95\% CI 1.07 to $1.61, \mathrm{p}=0.011)$. We also assessed the prognostic utility of troponin in ICI myocarditis. Troponin data were available for 138 of the 140 patients. Of these 138, 125 (90.6\%) had an elevated troponin. Of the 125 patients with elevated troponins, 68 (54.4\%) experienced a MACE, while of the 13 patients with normal troponins, only $1(7.7 \%)$ had a MACE ( $p=0.002)$. An elevated troponin was common and was not associated with increased MACE risk in the multivariable model (OR 2.54, 95\% CI 0.69 to $10.83, \mathrm{p}=0.17$ ).

Rates for each of the four MACE components (cardiovascular death, cardiac arrest, cardiogenic shock, and complete heart block) were higher among patients with a QRS duration $>110 \mathrm{~ms}$ than among those with a QRS duration $\leq 110 \mathrm{~ms}$ (figure 4 ). The most common MACE component in each was cardiovascular death $(16.2 \%$ among those with QRS $\leq 110 \mathrm{~ms}$, and $39.4 \%$ among those with QRS $>110 \mathrm{~ms}$ ).

\section{Association of QTc interval with MACE}

In contrast, a prolonged QTc-F interval was not associated with increased risk for MACE among men (HR 1.33, $95 \%$ CI 0.70 to $2.53, p=0.38$ ) or women (HR $1.48,95 \%$ CI 0.61 to $3.58, \mathrm{p}=0.39$ ) (figure $3 \mathrm{~B}, \mathrm{C}$ ). We also tested the association using QTc interval values corrected for heart rate using the Bazett formula. With this approach, among men, a QTc-B of $>450 \mathrm{~ms}$ was associated with an increased risk of MACE (HR 2.81, 95\% CI 1.42 to 5.38, p=0.002) (online supplemental file 5A). Among women, a QTc-B duration of $>460 \mathrm{~ms}$ was also associated with an increased risk of MACE (HR 3.00, 95\% CI 1.08 to 8.32 , log-rank $\mathrm{p}=0.03$ ) (online supplemental file $5 \mathrm{~B}$ ).

\section{DISCUSSION}

In this paper, we present novel findings describing increases in the QRS duration that may aid the diagnosis and risk-stratification of ICI myocarditis. This study has several unique and widely generalizable findings. First, we found that ICI myocarditis is associated with an unchanged PR interval but an increased QRS duration when compared with controls or cases at earlier timepoints (both prior to the initiation of ICI therapy and on ICI therapy but prior to the development of myocarditis). The QTc-F interval was prolonged compared with on-ICI controls but not on-ICI cases. Second, the increase in ECG intervals correlated with structural (LVEDV) and functional (EF) changes in the heart. Third, prolongations 
A

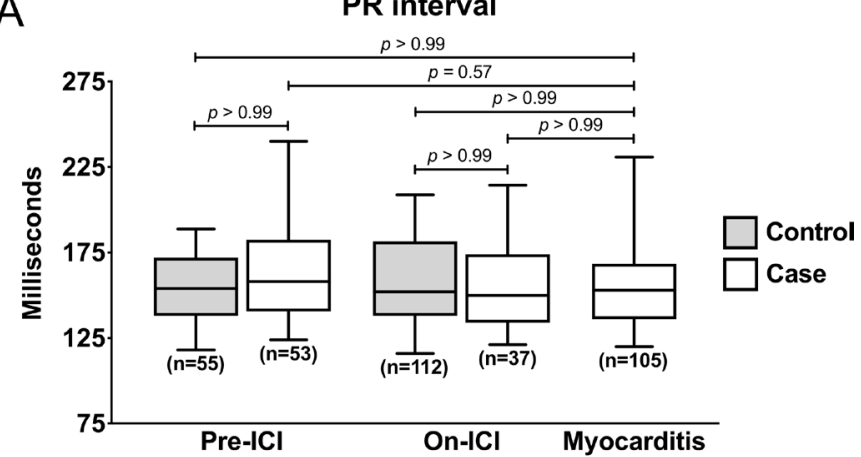

B

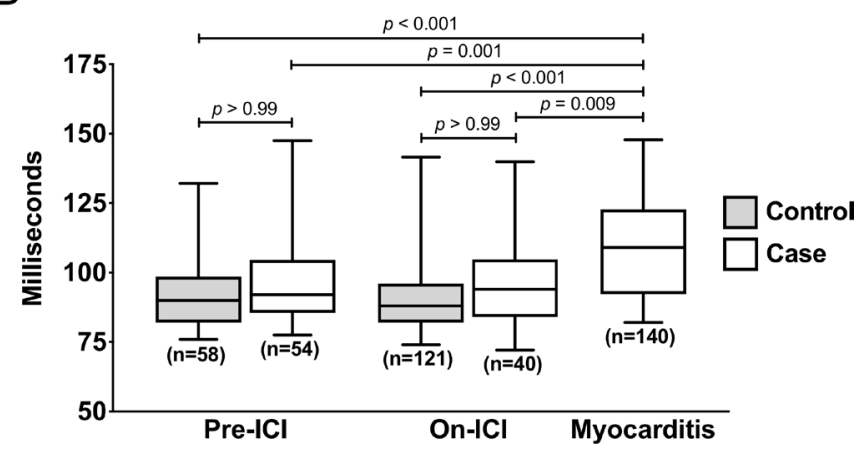

C

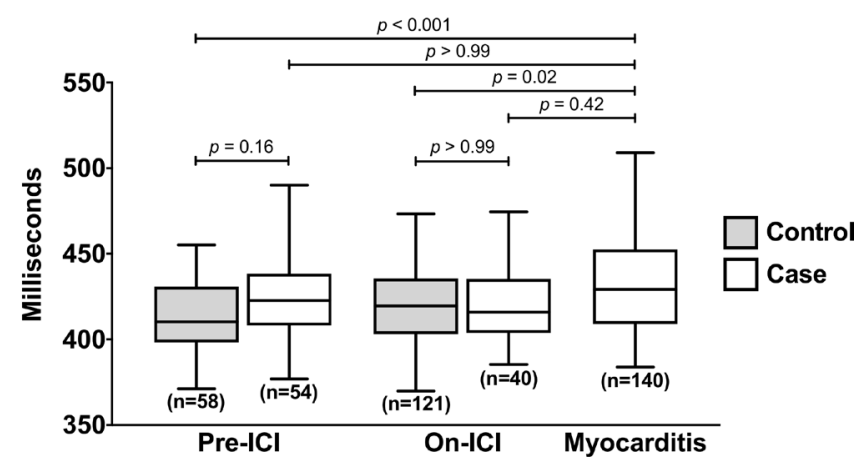

Figure 1 Changes in ECG parameters with ICl myocarditis. (A) PR interval, (B) QRS duration, and (C) QTC-F interval values were derived from ECGs obtained pre-ICl therapy ("baseline or pre-ICl"), after initiating ICI therapy ("on-ICl"), or at the time of myocarditis (for cases only; "myocarditis"). Shown are box-and-whisker plots with the central line indicating the median value, the margins of the box indicating the 25th/75th percentiles, and the whiskers indicating the 5th/95th percentiles. ICl, immune checkpoint inhibitor.

of QRS duration but not QTc-F interval at the time of myocarditis diagnosis were associated with an increased risk of MACE; specifically, a QRS duration $>110 \mathrm{~ms}$ was associated with a 3.28-fold increased risk of MACE. This association between prolonged QRS duration and MACE persisted after adjustment for several cardiovascular covariates. Fourth, each of the individual components of MACE occurred more frequently in myocarditis patients with a prolonged QRS duration, with cardiovascular death the most common.
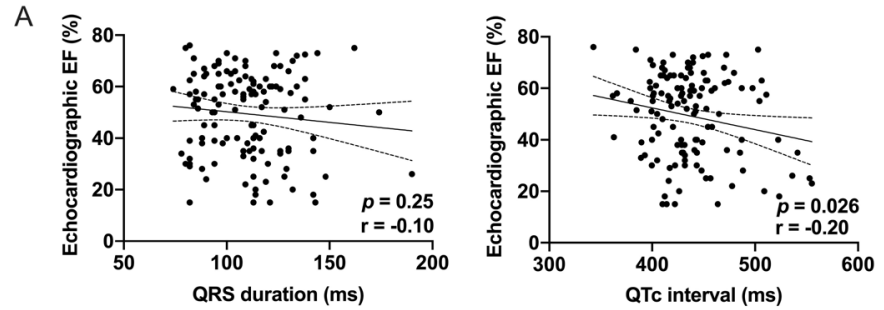

B
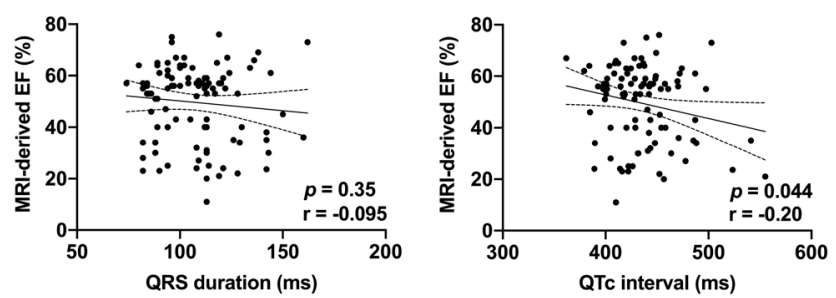

C
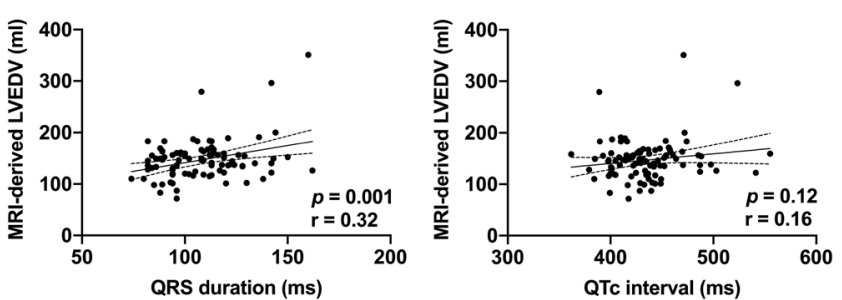

Figure 2 Relationship between ECG parameters, ejection fraction, and left ventricular volumes. (A) Scatter-plots of QRS duration (left) or QTc-F interval (right) versus echocardiographic left ventricular EF. Shown are the linear regression lines with their $95 \% \mathrm{Cl}$. (B) Scatter-plots of QRS duration (left) or QTc-F interval (right) versus EF. (C) Scatterplots of QRS duration (left) or QTc-F interval (right) versus LVEDV. EF, ejection fraction; LVEDV, left ventricular enddiastolic volume.

Despite its high morbidity, the diagnosis of ICI myocarditis can be challenging. ${ }^{2133-36}$ First, the presentation can be non-specific, both in terms of clinical symptoms and testing abnormalities. ${ }^{7132134-36}$ Second, the utility of standard cardiac testing in the diagnosis and risk-stratification of ICI myocarditis is not yet well established. ${ }^{21} 3536$ Serum troponin levels, typically a hallmark of myocardial injury/myocarditis, may be only modestly elevated, may be elevated for other reasons, or, in rare cases, even normal. ${ }^{713}$ Collectively, the available non-invasive diagnostic tests (including serum troponin levels, electrocardiography, and echocardiography) all fail to provide high specificity. ${ }^{41} 343537$ Third, the gold standard diagnostic test, endomyocardial biopsy, confers procedural risk that may be prohibitive for some patients and is not commonly performed. ${ }^{35} 38$ Our study now provides novel data that the QRS duration and QTc interval may be used as part of a diagnostic algorithm. However, changes in these ECG parameters can too have limited specificity, highlighting the fact that no test can be interpreted in isolation. Rather, surveillance of these values may be an effective and rapid means of identifying ICI recipients who should receive additional, more specific, cardiovascular testing. Prospective data will be needed to demonstrate the efficacy of this approach. 
A

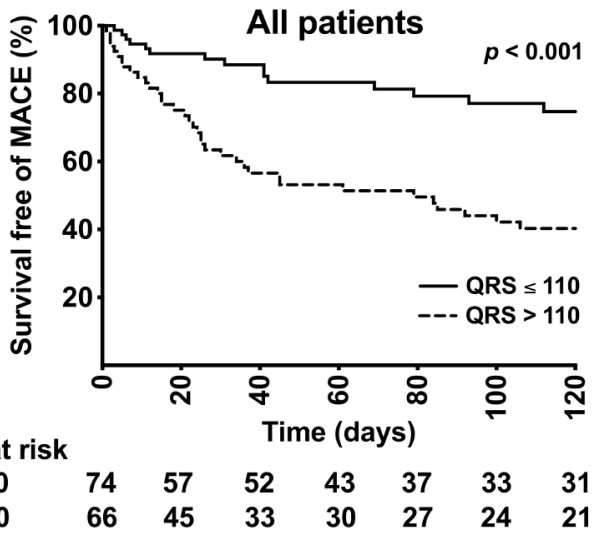

B

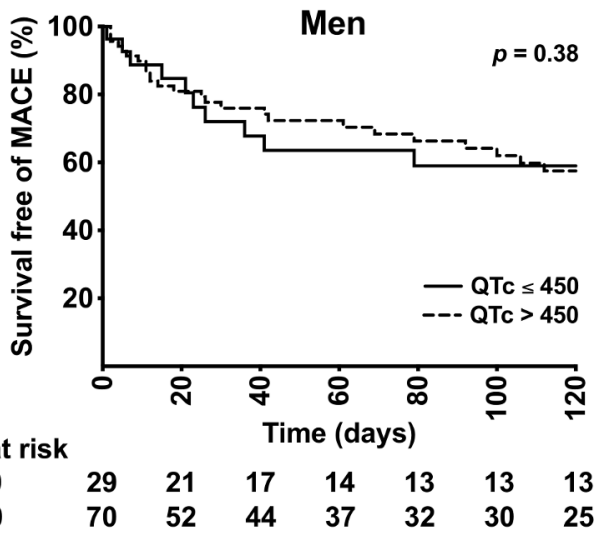

C

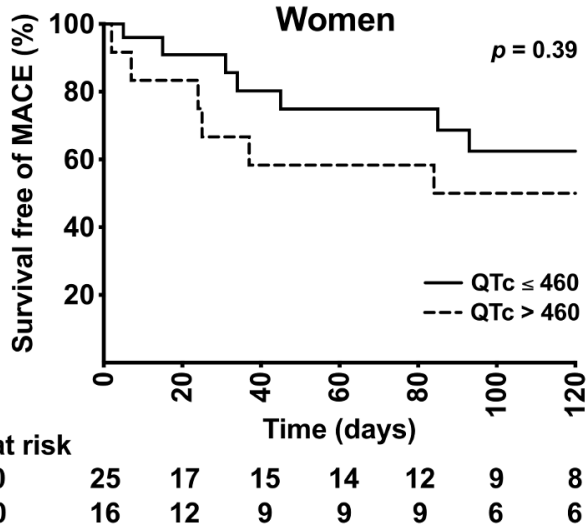

Figure 3 Association between QRS duration or QTc-F interval and MACE. (A) Kaplan-Meier curves indicate the occurrence of MACE over 120 days from time of diagnosis for myocarditis patients stratified by QRS duration. Similar analyses were performed for male (B) and female (C) myocarditis patients stratified by QTC-F interval as indicated. $P$ value obtained from the log-rank test. MACE, major adverse cardiac events.

Our study also suggests that the QRS duration may be used to risk-stratify ICI myocarditis patients. Prolongations of these values are associated with poor outcomes in a variety of conditions, including heart failure and acute myocardial infarction. ${ }^{39}$ The prognostic significance of a prolonged QRS duration or QTc interval in nonICI myocarditis is not clear. One study of patients with suspected myocarditis reported that a prolonged QRS
Table 2 Association with major adverse cardiac events (MACE) by multivariable analysis

\begin{tabular}{llll}
\hline Variable & OR & $95 \%$ Cl & P value \\
\hline QRS duration & 1.30 & 1.07 to 1.61 & 0.011 \\
\hline Age & 1.01 & 0.98 to 1.04 & 0.61 \\
Male sex & 0.55 & 0.21 to 1.40 & 0.22 \\
Hypertension & 0.62 & 0.24 to 1.55 & 0.31 \\
\hline Diabetes mellitus & 1.32 & 0.48 to 3.73 & 0.59 \\
Coronary artery disease & 0.34 & 0.07 to 1.36 & 0.14 \\
\hline Ejection fraction $<50 \%$ & 4.20 & 1.80 to 10.38 & 0.001 \\
\hline Elevated troponin & 2.54 & 0.69 to 10.83 & 0.17 \\
\hline
\end{tabular}

QRS duration was determined at the time of myocarditis diagnosis. For the QRS duration row, the OR reflects the MACE risk for an increase of $10 \mathrm{~ms}$ from the mean value across the myocarditis cohort. For the age row, the OR reflects the MACE risk for an increase of 1 year. For all other rows, the ORs reflect the MACE risk comparing those with the designated condition compared with those without. OR, odds ratio.

duration or QTc interval were each associated with poor outcomes, though only a prolonged QRS duration was found to be an independent predictor after adjustment for covariates. ${ }^{41}$ However, a more recent study of non-ICI myocarditis patients failed to find an association between QRS duration or QTc interval and adverse outcomes, though the sensitivity to detect an association was limited by a markedly lower adverse event rate. ${ }^{42}$ As background, ICI myocarditis has a higher adverse event rate compared with other irAEs or non-ICI lymphocytic myocarditis, and therefore the prognostic associations of prolonged ECG parameters may be more easily identified. ${ }^{3} 1415$

Cardiac inflammation alters intracardiac conduction patterns and can promote arrhythmogenesis. ${ }^{43}$ This may occur through several non-mutually exclusive mechanisms: (1) interplay between immune cells and cardiac fibroblasts and/or cardiomyocytes, leading to fibrosis ${ }^{45}$; (2) direct participation of immune cells, namely macrophages, in cardiac conduction ${ }^{46}$; and (3) the effects of

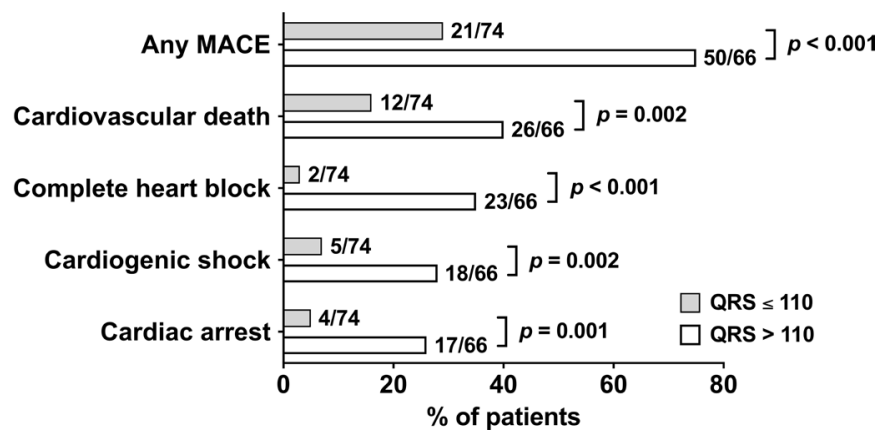

Figure 4 Frequency of MACE components. Frequencies of the indicated outcome among all myocarditis patients stratified by QRS duration are shown. Numbers at the end of each bar indicate the fraction of patients in each stratum with the indicated event. MACE, major adverse cardiac events. 
autoantibodies and cytokines on cardiomyocyte ion channels. ${ }^{47}$ In addition, patients with chronic inflammatory conditions have increased rates of conduction abnormalities, demonstrating that even inflammation outside the heart can affect cardiac conduction. ${ }^{48} 49$

In our analysis, the QT interval was corrected for heart rate using the Fridericia formula as this is more accurate than the commonly used Bazett formula and considered the most appropriate strategy by the US Food and Drug Administration. ${ }^{22-24} 50$ The Bazett formula, however, remains frequently used in clinical practice, so we performed the same analysis using the Bazett-corrected QTc interval. With these values, an association between QTc-B interval and MACE was observed for both men and women, suggesting a potential role for the Bazettcorrected QTc interval towards ICI myocarditis risk stratification.

These findings should be interpreted within the overall limitations of the retrospective study design. First, the clinical data collection was not protocolized and was instead derived from medical records at each participating center, which in some cases led to incomplete data. Second, the timing of ECGs relative to ICI therapy initiation was not standardized, thereby leading to variability within each cohort. Third, we identified differences in the use of antiPD-1 agents, anti-PD-L1 agents, and combination therapy between the control and myocarditis groups which may have confounded our results. Fourth, all controls were derived from a single center.

In summary, we observed prolongation of the QRS duration in the setting of ICI myocarditis. A QRS duration, but not QTc-F interval, greater than normal was associated with increased risk of MACE. These findings illustrate the potential diagnostic and prognostic value of easily obtained ECG parameters in ICI myocarditis.

\section{Author affiliations}

${ }^{1}$ Cardio-Oncology Program, Division of Cardiology, Department of Medicine, Massachusetts General Hospital, Boston, Massachusetts, USA

${ }^{2}$ Cardiovascular Imaging Research Center (CIRC), Division of Cardiology and Department of Radiology, Massachusetts General Hospital, Boston, Massachusetts, USA

${ }^{3}$ Cardio-Oncology Program, Cardiology Division, NewYork-Presbyterian Hospital, Weill Cornell Medicine, New York, New York, USA

${ }^{4}$ Cardio-Oncology Program, Division of Cardiology, Department of Medicine, Montefiore Medical Center, Albert Einstein College of Medicine, Bronx, New York, USA

${ }^{5}$ Cardiology Division, Memorial Sloan Kettering Cancer Center, Weill Cornell Medicine, New York, New York, USA

${ }^{6}$ Cardio-Oncology Program, Division of Cardiology, Hopitaux Universitaires Est Parisien, Paris, France

${ }^{7}$ Cardio-Oncology Program, MedStar Heart and Vascular Institute, MedStar Washington Hospital Center, Washington, DC, USA

${ }^{8}$ Cardio-Oncology Program, Division of Cardiovascular Medicine, H. Lee Moffitt Cancer Center \& Research Institute and University of South Florida, Tampa, Florida, USA

${ }^{9}$ Department of Dermatology, University Hospital Erlangen, Friedrich-AlexanderUniversity Erlangen-Nürnberg (FAU), Erlangen, Germany

${ }^{10}$ Discipline of Cardiology, Department of Medicine, Faculty of Medical Science, State University of Campinas, Campinas, Brazil

${ }^{11}$ Division of Oncology and Hematology, Department of Medicine, Lehigh Valley Hospital, Allentown, Pennsylvania, USA
${ }^{12}$ Cardiology Division, Mount Sinai Medical Center, New York, New York, USA ${ }^{13}$ Cardio-Oncology Program, Department of Hematology and Medical Oncology, Winship Cancer Institute, Emory University School of Medicine, Atlanta, Georgia, USA

${ }^{14}$ Department of Translational Medical Sciences, Interdepartmental Center of Clinical and Translational Research (CIRCET), Interdepartmental Hypertension Research Center (CIRIAPA), Università degli Studi di Napoli Federico II, Naples, Italy

${ }^{15}$ Cardiology Department, Hospital General Universitario Gregorio Marañón, Centro de Investigación Biomédica en Red CardioVascular (CIBER-CV), Madrid, Spain

${ }^{16}$ Faculty of Medicine, University of Southampton, Southampton, UK

${ }^{17}$ Massachusetts General Hospital Cancer Center, Department of Medicine,

Massachusetts General Hospital, Boston, Massachusetts, USA

${ }^{18}$ Cardio-Oncology Program, Division of Cardiovascular Medicine, Lahey Hospital and Medical Center, Burlington, Massachusetts, USA

${ }^{19}$ UCLA Cardio-Oncology Program, Division of Cardiology, Department of Medicine, University of California at Los Angeles, Los Angeles, California, USA

${ }^{20}$ Department of Dermatology and Allergy, LMU Klinikum, Munich, Germany

${ }^{21}$ Mediterranean University Center of Cardio-Oncology, Aix-Marseille University, North Hospital, Marseille, France

${ }^{22}$ Abramson Cancer Center, Perelman School of Medicine, University of

Pennsylvania, Philadelphia, Pennsylvania, USA

${ }^{23}$ Cardio-Oncology Service, Royal Brompton Hospital and Imperial College London, London, UK

${ }^{24}$ Cardio-Oncology Program, Division of Cardiology, Department of Medicine, Brigham and Women's Hospital, Boston, Massachusetts, USA

${ }^{25}$ Ted Rogers Program in Cardiotoxicity Prevention, Peter Munk Cardiac Center, Division of Cardiology, Toronto General Hospital, University of Toronto, Toronto, Ontario, Canada

Twitter Amna Zafar @AZafar_MD, Dahlia Banerji @DahliaBanerjiMD, Eduardo Zatarain-Nicolás @EduZatarain, Franck Thuny @franckthuny and Tomas G Neilan @ TomasNeilan

Contributors DZ devised the project idea, designed the study, performed statistical analysis, and wrote the manuscript. MZOH and RMA performed statistical analysis. MA, AZ, LZh, DB, MJ-O'C, SPM, RS, LZu, KLR, and JC provided critical intellectual input to study design and analysis. SSM created the registry of myocarditis cases. SH and HKG provided both administrative support as well as collection of data. CLC, SE, AB, MA, BJF, MCK, ORC-F, MAR, GS, AM, CT, EZ, MM, DG, RS, SG, EHY, LH, FT, ARL, JG, PT, MGF, and AN provided critical intellectual input to study design and analysis and provided data on clinical myocarditis cases. TGN created the registry of myocarditis cases, devised the project idea, designed the study, supervised the statistical analysis and manuscript development, and provided funding for the project.

Funding This work was supported by the National Institutes of Health (P30CA008748 to DG and CLC; R01HL137562, R01HL130539, and K24HL150238 to TGN; and T32HL007208-39 to DAZ).

Disclaimer The study funders had no role in the design of the study; the collection, analysis, and interpretation of the data; the writing of the manuscript; and the decision to submit the manuscript for publication.

Competing interests SSM has received consultancy fees from OMR Globus, Alpha Detail, and Opinion Research Team. RS has been a consultant to Merck and Novartis. LH has received consultancy, advisory board, and speaker fees from MSD, BMS, Roche, Novartis, Amgen, and Curevac. LZu has been a consultant to Merck. JG has received research support from Amgen. AN has received research support from Amgen and has been a consultant for Takeda Oncology and AstraZeneca. TGN has received advisory fees from Parexel, AbbVie, H3-Biomedicine, Aprea Therapeutics, BMS, and Intrinsic Imaging. TGN has received grant funding from AstraZeneca.

Patient consent for publication Not required.

Ethics approval The institutional review board at each center approved the study, and the requirement for written informed consent was waived.

Provenance and peer review Not commissioned; externally peer reviewed.

Data availability statement Data are available upon reasonable request.

Supplemental material This content has been supplied by the author(s). It has not been vetted by BMJ Publishing Group Limited (BMJ) and may not have been peer-reviewed. Any opinions or recommendations discussed are solely those of the author(s) and are not endorsed by BMJ. BMJ disclaims all liability and 
responsibility arising from any reliance placed on the content. Where the content includes any translated material, BMJ does not warrant the accuracy and reliability of the translations (including but not limited to local regulations, clinical guidelines, terminology, drug names and drug dosages), and is not responsible for any error and/or omissions arising from translation and adaptation or otherwise.

Open access This is an open access article distributed in accordance with the Creative Commons Attribution Non Commercial (CC BY-NC 4.0) license, which permits others to distribute, remix, adapt, build upon this work non-commercially, and license their derivative works on different terms, provided the original work is properly cited, appropriate credit is given, any changes made indicated, and the use is non-commercial. See http://creativecommons.org/licenses/by-nc/4.0/.

\section{ORCID iDs}

Daniel A Zlotoff http://orcid.org/0000-0001-9007-7295

Franck Thuny http://orcid.org/0000-0002-8727-7154

\section{REFERENCES}

1 Topalian SL, Drake CG, Pardoll DM. Immune checkpoint blockade: a common denominator approach to cancer therapy. Cancer Cell 2015;27:450-61.

2 Postow MA, Sidlow R, Hellmann MD. Immune-Related adverse events associated with immune checkpoint blockade. N Engl J Med 2018;378:158-68.

3 Wang DY, Salem J-E, Cohen JV, et al. Fatal toxic effects associated with immune checkpoint inhibitors: a systematic review and metaanalysis. JAMA Oncol 2018;4:1721-8.

$4 \mathrm{Hu}$ J-R, Florido R, Lipson EJ, et al. Cardiovascular toxicities associated with immune checkpoint inhibitors. Cardiovasc Res 2019;115:854-68.

5 Awadalla M, Golden DLA, Mahmood SS, et al. Influenza vaccination and myocarditis among patients receiving immune checkpoint inhibitors. J Immunother Cancer 2019;7:53.

6 Salem J-E, Manouchehri A, Moey M, et al. Cardiovascular toxicities associated with immune checkpoint inhibitors: an observational, retrospective, pharmacovigilance study. Lancet Oncol 2018;19:1579-89.

7 Mahmood SS, Fradley MG, Cohen JV, et al. Myocarditis in patients treated with immune checkpoint inhibitors. J Am Coll Cardiol 2018;71:1755-64.

8 Zhang L, Jones-O'Connor M, Awadalla M, et al. Cardiotoxicity of immune checkpoint inhibitors. Curr Treat Options Cardiovasc Med 2019;21:32.

9 Ganatra S, Neilan TG. Immune checkpoint inhibitor-associated myocarditis. Oncologist 2018;23:879-86.

10 Johnson DB, Balko JM, Compton ML, et al. Fulminant myocarditis with combination immune checkpoint blockade. N Engl J Med 2016;375:1749-55

11 Drobni ZD, Alvi RM, Taron J, et al. Association between immune checkpoint inhibitors with cardiovascular events and atherosclerotic plaque. Circulation 2020;142:2299-311.

12 Ederhy S, Cautela J, Ancedy Y, et al. Takotsubo-Like Syndrome in Cancer Patients Treated With Immune Checkpoint Inhibitors. JACC Cardiovasc Imaging 2018;11:1187-90.

13 Escudier M, Cautela J, Malissen N, et al. Clinical features, management, and outcomes of immune checkpoint Inhibitor-Related cardiotoxicity. Circulation 2017;136:2085-7.

14 Grün S, Schumm J, Greulich S, et al. Long-term follow-up of biopsyproven viral myocarditis: predictors of mortality and incomplete recovery. J Am Coll Cardiol 2012;59:1604-15.

15 Gräni C, Eichhorn C, Bière L, et al. Prognostic Value of Cardiac Magnetic Resonance Tissue Characterization in Risk Stratifying Patients With Suspected Myocarditis. J Am Coll Cardiol 2017;70:1964-76.

16 Neilan TG, Rothenberg ML, Amiri-Kordestani L, et al. Myocarditis associated with immune checkpoint inhibitors: an expert consensus on data gaps and a call to action. Oncologist 2018;23:874-8.

17 Alvi RM, Frigault MJ, Fradley MG, et al. Cardiovascular events among adults treated with chimeric antigen receptor T-cells (CAR-T). J Am Coll Cardiol 2019;74:3099-108.

18 Heymans S, Eriksson U, Lehtonen J, et al. The Quest for New Approaches in Myocarditis and Inflammatory Cardiomyopathy. J Am Coll Cardiol 2016;68:2348-64.

19 Sagar S, Liu PP, Cooper LT. Myocarditis. Lancet 2012;379:738-47.

20 Porta-Sánchez A, Gilbert C, Spears D, et al. Incidence, diagnosis, and management of QT prolongation induced by cancer therapies: a systematic review. J Am Heart Assoc 2017;6. doi:10.1161/ JAHA.117.007724. [Epub ahead of print: 07 Dec 2017].
21 Awadalla M, Mahmood SS, Groarke JD, et al. Global longitudinal strain and cardiac events in patients with immune checkpoint Inhibitor-Related myocarditis. J Am Coll Cardiol 2020;75:467-78.

22 Fridericia LS. The duration of systole in an electrocardiogram in normal humans and in patients with heart disease. 1920. Ann Noninvasive Electrocardiol 2003;8:343-51.

23 Muluneh B, Richardson DR, Hicks C, et al. Trials and tribulations of corrected QT interval monitoring in oncology: rationale for a Practice-Changing standardized approach. J Clin Oncol 2019;37:2719-21.

24 US Food and Drug Administration. E14 clinical evaluation of QT/ QTc interval prolongation and proarrhythmic potential for NonAntiarrhythmic drugs - questions and answers (R3), 2017. Available: https://www.fda.gov/media/71379/download

25 Surawicz B, Childers R, Deal BJ, et al. AHA/ACCF/HRS recommendations for the standardization and interpretation of the electrocardiogram: Part III: intraventricular conduction disturbances: a scientific statement from the American heart association electrocardiography and arrhythmias Committee, Council on clinical cardiology; the American College of cardiology Foundation; and the heart rhythm Society. endorsed by the International Society for computerized Electrocardiology. J Am Coll Cardiol 2009;53:976-81.

26 Rautaharju PM, Surawicz B, Gettes LS, et al. AHA/ACCF/HRS recommendations for the standardization and interpretation of the electrocardiogram: Part IV: the ST segment, T and U waves, and the QT interval: a scientific statement from the American heart association electrocardiography and arrhythmias Committee, Council on clinical cardiology; the American College of cardiology Foundation; and the heart rhythm Society. endorsed by the International Society for computerized Electrocardiology. J Am Coll Cardiol 2009;53:982-91.

27 Caforio ALP, Pankuweit S, Arbustini E, et al. Current state of knowledge on aetiology, diagnosis, management, and therapy of myocarditis: a position statement of the European Society of cardiology Working group on myocardial and pericardial diseases. Eur Heart J 2013;34:2636-48. 48a-48d.

28 Ammirati E, Cipriani M, Moro C, et al. Clinical presentation and outcome in a contemporary cohort of patients with acute myocarditis: multicenter Lombardy registry. Circulation 2018;138:1088-99.

29 Hicks KA, Tcheng JE, Bozkurt B, et al. 2014 ACC/AHA key data elements and definitions for cardiovascular endpoint events in clinical trials: a report of the American College of Cardiology/ American heart association Task force on clinical data standards (writing Committee to develop cardiovascular endpoints data standards). J Am Coll Cardiol 2015;66:403-69.

30 Neilan TG, Farhad H, Mayrhofer T, et al. Late gadolinium enhancement among survivors of sudden cardiac arrest. JACC Cardiovasc Imaging 2015;8:414-23.

31 Reynolds HR, Hochman JS. Cardiogenic shock: current concepts and improving outcomes. Circulation 2008;117:686-97.

32 Therneau TM, Grambsch PM. Proportional hazards tests and diagnostics based on weighted residuals. Biometrika 1994;81:515-26.

33 Trachtenberg B, Hussain F, Mukherjee A. Immune checkpoint Inhibitor-Related cardiotoxicity. Methodist Debakey Cardiovasc J 2018;14:e1-4.

34 Zhang L, Zlotoff DA, Awadalla M, et al. Major adverse cardiovascular events and the timing and dose of corticosteroids in immune checkpoint inhibitor-associated myocarditis. Circulation 2020;141:2031-4.

35 Zhang L, Awadalla M, Mahmood SS, et al. Cardiovascular magnetic resonance in immune checkpoint inhibitor-associated myocarditis. Eur Heart J 2020;41:1733-43.

36 Norwood TG, Westbrook BC, Johnson DB, et al. Smoldering myocarditis following immune checkpoint blockade. J Immunother Cancer 2017:5:91.

37 Sarocchi M, Grossi F, Arboscello E, et al. Serial troponin for early detection of nivolumab cardiotoxicity in advanced non-small cell lung cancer patients. Oncologist 2018;23:936-42.

38 Shah M, Tayar JH, Abdel-Wahab N, et al. Myositis as an adverse event of immune checkpoint blockade for cancer therapy. Semin Arthritis Rheum 2019;48:736-40.

39 Lund LH, Jurga J, Edner M, et al. Prevalence, correlates, and prognostic significance of QRS prolongation in heart failure with reduced and preserved ejection fraction. Eur Heart $J$ 2013;34:529-39.

40 Yerra L, Anavekar N, Skali H, et al. Association of QRS duration and outcomes after myocardial infarction: the VALIANT trial. Heart Rhythm 2006;3:313-6. 
41 Ukena C, Mahfoud F, Kindermann I, et al. Prognostic electrocardiographic parameters in patients with suspected myocarditis. Eur J Heart Fail 2011;13:398-405.

42 Chen S, Hoss S, Zeniou V, et al. Electrocardiographic predictors of morbidity and mortality in patients with acute myocarditis: the importance of QRS-T angle. J Card Fail 2018;24:3-8.

43 Swirski FK, Nahrendorf M. Cardioimmunology: the immune system in cardiac homeostasis and disease. Nat Rev Immunol 2018;18:733-44.

44 Lazzerini PE, Laghi-Pasini F, Boutjdir M, et al. Cardioimmunology of arrhythmias: the role of autoimmune and inflammatory cardiac channelopathies. Nat Rev Immunol 2019;19:63-4.

45 Suthahar N, Meijers WC, Silljé HHW, et al. From inflammation to Fibrosis-Molecular and cellular mechanisms of myocardial tissue remodelling and perspectives on differential treatment opportunities. Curr Heart Fail Rep 2017;14:235-50.
46 Hulsmans M, Clauss S, Xiao L, et al. Macrophages facilitate electrical conduction in the heart. Cell 2017;169:510-22. e20.

47 Lazzerini PE, Capecchi PL, Laghi-Pasini F, et al. Autoimmune channelopathies as a novel mechanism in cardiac arrhythmias. Nat Rev Cardiol 2017;14:521-35.

48 Seferović PM, Ristić AD, Maksimović R, et al. Cardiac arrhythmias and conduction disturbances in autoimmune rheumatic diseases. Rheumatology 2006;45 Suppl 4:iv39-42.

49 Prasad M, Hermann J, Gabriel SE, et al. Cardiorheumatology: cardiac involvement in systemic rheumatic disease. Nat Rev Cardiol 2015;12:168-76.

50 Bazett HC. An analysis of the time-relations of electrocardiograms. Heart 1920;7:353-70. 